\title{
Designing a Mark-Recapture Study to Allow for Local Emigration
}

\begin{abstract}
David Fletcher, Stephen DAwson, and Elisabeth SLOOTEN
In using a population projection model to help manage the conservation of a longlived species, we usually need a reliable estimate of adult survival. Mark-recapture studies are often used to estimate survival, and typically require the assumption that there is no permanent emigration from the study area. We consider how such a study might be extended to allow for local emigration, that is, movement of individuals into an area peripheral to the study area. In particular, we focus on the question as to how much field effort is required in this peripheral area in order to obtain sufficiently precise estimates of both the survival probability and the probability of local emigration. We consider the use of multi-state, mark-recapture models as a means of providing these estimates and show how to assess the precision of a potential study design by calculating the expected confidence limits associated with the resulting estimates. We consider a range of design scenarios for the situation that motivated this work, involving a population of Hector's dolphins in New Zealand. For this case, it appears that there is little gain in precision once a capture probability of 0.4 is reached in the peripheral area.
\end{abstract}

Key Words: Capture; Multi-state model; Profile likelihood interval; Survival

\section{INTRODUCTION}

In conservation biology, population projection models are frequently used to evaluate the potential effects of alternative management actions. For long-lived species, the most important parameter to estimate reliably is adult survival. In most studies, there is no way of discriminating between death and emigration, potentially making the estimate of "survival" pessimistic. Usually, emigration is assumed to be negligible, even though a low level may have an impact on population projections. For example, Heppel, Caswell, and Crowder (2000) considered a widely applicable age-structured model in which there are $\alpha-1$ juvenile stages and one adult stage, and in which both adult survival and fertility are assumed to be

David Fletcher is Senior Lecturer, Department of Mathematics and Statistics, University of Otago, P.O. Box 56, Dunedin, New Zealand (E-mail: dfletcher@maths.otago.ac.nz). Stephen Dawson is Senior Lecturer, Department of Marine Science, University of Otago, Dunedin, New Zealand. Elisabeth Slooten is Senior Lecturer and Director, Environmental Science, University of Otago, Dunedin, New Zealand.

(c)2002 American Statistical Association and the International Biometric Society

Journal of Agricultural, Biological, and Environmental Statistics, Volume 7, Number 4, Pages 1-8 DOI: $10.1198 / 108571102799$ 
independent of age. They showed that for this type of model the elasticity for adult survival (proportional effect on population growth rate $\lambda$ of a proportional change in adult survival $P)$ can be written as

$$
e_{P}=\frac{1}{1+\alpha\left(\frac{\lambda}{P}-1\right)} .
$$

For the population we consider later, sensible working values are $\lambda=1, \alpha=8$, and $P=0.9$. The above equation then gives $e_{P}=0.53$. If the probability of local emigration is 0.05 (say), we would have a relative bias in $P$ of -0.05 and therefore be underestimating $\lambda$ by $\left(e_{P} \times 0.05\right) \times \lambda=(0.53 \times 0.05) \times 1=0.027$.

In this article we attempt to allow for emigration by extending field effort into an area peripheral to the main study area. Movement of animals from the main area to this peripheral area we call local emigration. If the extra field effort leads to an estimate of local emigration that is small enough to have little impact on population projections, it might be argued that emigration beyond this periphery will also be small enough to ignore. In addition, a measure of local emigration can be of use for other management purposes, such as deciding upon an appropriate size for a protected area.

Multi-state models for mark-recapture have been used by a number of researchers to study animal population dynamics (Arnason 1972, 1973; Brownie et al. 1993; Cam et al. 1998; Nichols and Kendall 1995; Schwartz, Schweigert, and Arnason 1993; Scofield, Fletcher, and Robertson 2001), and as a means of allowing for capture heterogeneity (Cameron et al. 1999). The term state is used to describe a characteristic such as breeding status (Cam et al. 1998; Scofield et al. 2001) and geographical area (Arnason 1973; Brownie et al. 1993; Schwartz et al. 1993). These models allow estimation of three types of parameter: the survival and capture probabilities for each state, as well as the probability of transition between states. These parameters can be time-dependent or independent, and can also be related to covariates. Of interest here is the fact that such a model can be used to estimate movement probabilities between geographical areas. If we consider the peripheral area to be separate from the main study area, this type of model can therefore be used to estimate the amount of local emigration. In addition, it also provides an estimate of survival that allows for any such emigration.

Suppose we want to ensure that our study is able to provide reasonably precise estimates of both the survival probability and the probability of local emigration. How much field effort do we need in the peripheral area? Here we present a method for answering this question, and illustrate its use in the context of a study of Hector's dolphin.

\section{METHOD}

We address the above question by considering the effect of study design on the $95 \%$ confidence limits for both the survival probability and the probability of local emigration. We do this by generating an "expected" multi-state $m$-array and then performing the multi-state 
analysis, in a manner akin to the general approach described by Burnham et al. (1987). This analysis can be used to provide confidence limits that correspond to the "true" standard errors (asymptotically) and therefore provide an indication of the precision of the corresponding parameter estimates. We calculated confidence limits using profile likelihood (Lebreton, Burnham, Clobert, and Anderson 1992; McCullagh and Nelder 1989), and performed all calculations in an Excel spreadsheet, a copy of which is available from the first author.

Details of the likelihood function for multi-state models are provided in Brownie et al. (1993) and Cameron et al. (1999). The expected multi-state $m$-array can be generated as follows, using the notation in Table 3 of Cameron et al. (1999). If $N_{i}^{r}$ is the number of individuals in area $r$ at time $i$, then, $E\left(R_{i}^{r}\right)=N_{i}^{r} p_{i}^{r}$ and $E\left(m_{i j}^{r s}\right)=E\left(R_{i}^{r}\right) \Pi_{i j}^{r s}(r=$ $1,2 ; i=1, \ldots, k-1 ; j=i+1, \ldots, k)$. In order to specify the $N_{i}^{r}$, we assume that $N_{i}^{1}+N_{i}^{2}$ is the same for each sampling occasion $i$. Given the initial population sizes in each area, a natural way to ensure this is to set $N_{i+1}^{r}=a_{i}^{1 r} N_{i}^{1}+a_{i}^{2 r} N_{i}^{2}(r=1,2 ; i=2, \ldots, k)$.

In the actual analysis, it would be appropriate to fit and compare a variety of multi-state models using, for example, the program MARK (White and Burnham 1999). For the purpose of assessing potential designs, we consider a simple model, in which every parameter is considered to be time-independent. This model involves six parameters: the survival and capture probabilities in both areas, and the movement probabilities between them.

We now illustrate our approach by considering the management situation that motivated this work.

\section{HECTOR'S DOLPHIN}

Hector's dolphin (Cephalorhynchus hectori) is a small, coastal delphinid found solely in the inshore waters of New Zealand. The species has been studied extensively around Banks Peninsula (Figure 1), where a sanctuary free of commercial gillnetting was established in 1988 (Dawson and Slooten 1993). In order to help assess the status of the population, a longterm photo-ID survey of individually identifiable dolphins was set up in part to estimate adult survival probability at Banks Peninsula (Slooten, Dawson, and Lad 1992; Cameron et al. 1999). Here we consider the degree to which that study would need to be extended in order to obtain precise estimates of movement to either the north or south of the Banks Peninsula sanctuary, these two areas being treated as one peripheral area (Figure 1).

It is important to note that this example is different from many mark-recapture studies in that only the individually identifiable dolphins provide any data. Marking occurs naturally, and is not carried out by the researcher. In all that follows, we therefore use the term "individuals" to refer only to dolphins that are individually identifiable. Similarly, "population size" refers to the total number of individually identifiable dolphins.

Suppose we are interested in the degree of precision that we expect to achieve with a study over ten years, where the survival probability in both areas is assumed to be approximately 0.9. We suspect that the probability of local emigration (movement from the main area to the peripheral area) is around 0.05 , and that there is no movement in the reverse direction. Finally, suppose there are approximately 100 individuals in the main area and 10 


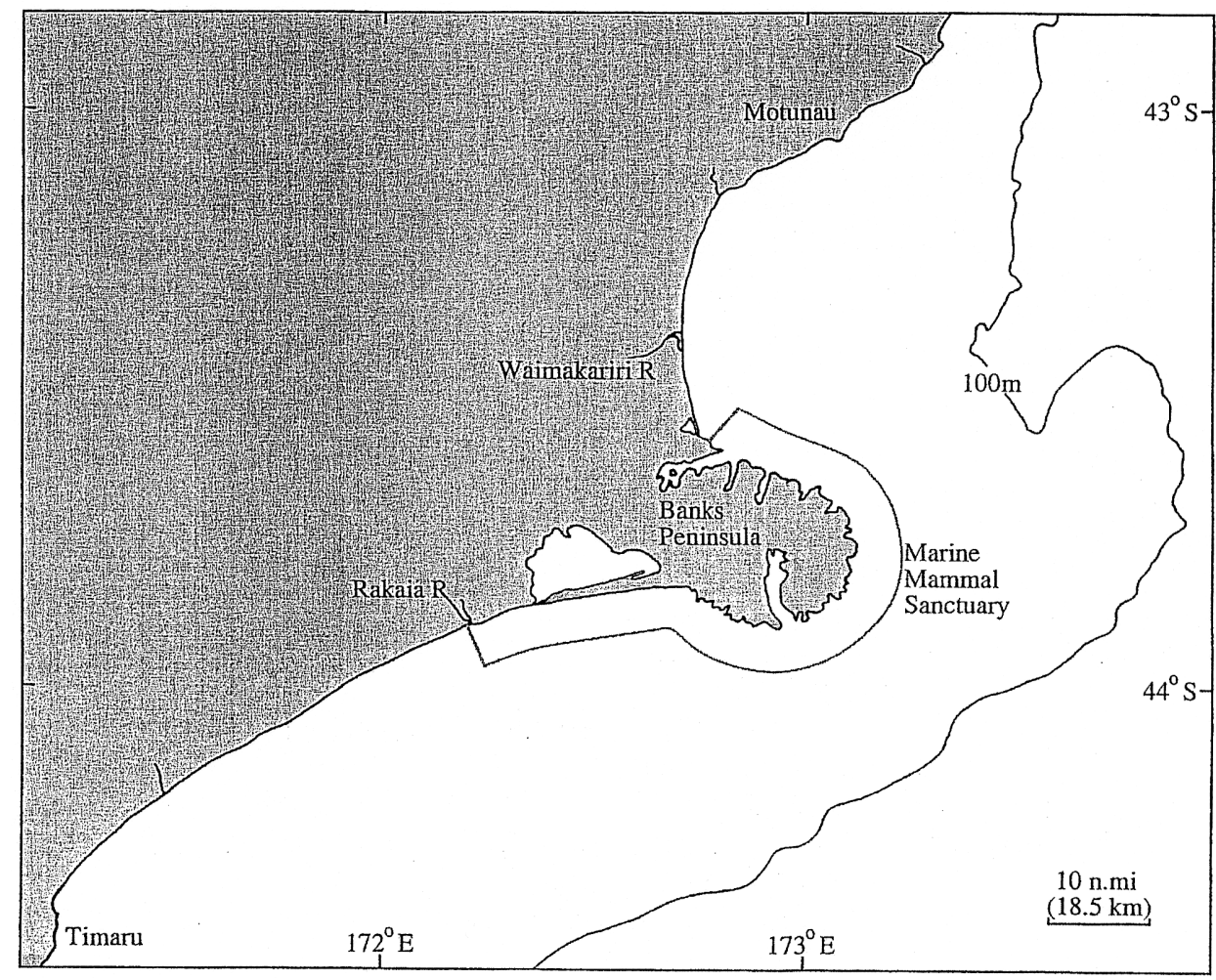

Figure 1. Hector's dolphin study areas. The main study area is the marine mammal sanctuary. The peripheral study area is from the northern boundary of the sanctuary to Motunau and from the southern boundary of the sanctuary to Timaru.

in the peripheral area.

How much field effort do we need in each area? In order to obtain a reliable estimate of survival for the main area, we envisage aiming for a capture probability in that area of 0.5 (see Pollock, Nichols, Brownie, and Hines 1990). This would correspond to an expected capture of 50 individuals in that area in the first year. Suppose we also consider trying to achieve a capture probability of 0.5 in the peripheral area, corresponding to an expected capture of five individuals in that area in the first year.

The expected $m$-array corresponding to this scenario is shown in Table 1. If we use a time-independent multi-state model as described earlier, and set the survival probability in the two areas to be equal, the estimate of survival is 0.90 , with $95 \%$ confidence limits of 0.83 and 0.96 . We can compare this to an equivalent scenario in which there is no field effort in the peripheral area by generating an expected $m$-array with the capture probability in the peripheral area set to zero. Analysis of the values in this $m$-array for the main area, using a single-state time-independent model, gives an estimate of survival of 0.86 , with $95 \%$ confidence limits of 0.82 and 0.89 . Note that the estimate is what we would expect, namely the true survival probability multiplied by the probability of not emigrating $(0.9 \times 0.95=0.855)$. This change in the estimate of the survival probability could alter 
Table 1. Expected Multi-state $m$-array, Showing the Values of $E\left(R_{i}^{r}\right)$ and $E\left(m_{i j}^{r s}\right)$, for a Ten-Year Study. Survival probability $=0.9$ in both areas, capture probability $=0.5$ in both areas, probability of local emigration $=0.05$, initial population size in area $1=100$, initial population size in area $2=10$. All expected frequencies are shown to one decimal place.

\begin{tabular}{|c|c|c|c|c|c|c|c|c|c|c|c|c|c|c|c|c|c|c|c|c|}
\hline \multirow[b]{3}{*}{ Year } & \multirow[b]{3}{*}{ Area } & \multirow{3}{*}{$\begin{array}{l}\text { Number } \\
\text { captured }\end{array}$} & \multicolumn{18}{|c|}{ Year and area of first resighting } \\
\hline & & & \multicolumn{2}{|c|}{2} & \multicolumn{2}{|c|}{3} & \multicolumn{2}{|c|}{4} & \multicolumn{2}{|l|}{5} & \multicolumn{2}{|l|}{6} & \multicolumn{2}{|c|}{7} & \multicolumn{2}{|c|}{8} & \multicolumn{2}{|c|}{9} & \multicolumn{2}{|c|}{10} \\
\hline & & & 1 & 2 & 1 & 2 & 1 & 2 & 1 & 2 & 1 & 2 & 1 & 2 & 1 & 2 & 1 & 2 & 1 & 2 \\
\hline \multirow[t]{2}{*}{1} & 1 & 50.0 & 21.4 & 1.1 & 9.1 & 1.0 & 3.9 & 0.6 & 1.7 & 0.4 & 0.7 & 0.2 & 0.3 & 0.1 & 0.1 & 0.1 & 0.1 & 0.0 & 0.0 & 0.0 \\
\hline & 2 & 5.0 & 0.0 & 2.3 & 0.0 & 1.0 & 0.0 & 0.5 & 0.0 & 0.2 & 0.0 & 0.1 & 0.0 & 0.0 & 0.0 & 0.0 & 0.0 & 0.0 & 0.0 & 0.0 \\
\hline \multirow[t]{2}{*}{2} & 1 & 47.5 & & & 20.3 & 1.1 & 8.7 & 0.9 & 3.7 & 0.6 & 1.6 & 0.4 & 0.7 & 0.2 & 0.3 & 0.1 & 0.1 & 0.1 & 0.1 & 0.0 \\
\hline & 2 & 7.5 & & & 0.0 & 3.4 & 0.0 & 1.5 & 0.0 & 0.7 & 0.0 & 0.3 & 0.0 & 0.1 & 0.0 & 0.1 & 0.0 & 0.0 & 0.0 & 0.0 \\
\hline \multirow[t]{2}{*}{3} & 1 & 45.1 & & & & & 19.3 & 1.0 & 8.2 & 0.9 & 3.5 & 0.6 & 1.5 & 0.3 & 0.6 & 0.2 & 0.3 & 0.1 & 0.1 & 0.1 \\
\hline & 2 & 9.9 & & & & & 0.0 & 4.4 & 0.0 & 2.0 & 0.0 & 0.9 & 0.0 & 0.4 & 0.0 & 0.2 & 0.0 & 0.1 & 0.0 & 0.0 \\
\hline \multirow[t]{2}{*}{4} & 1 & 42.9 & & & & & & & 18.3 & 1.0 & 7.8 & 0.8 & 3.3 & 0.6 & 1.4 & 0.3 & 0.6 & 0.2 & 0.3 & 0.1 \\
\hline & 2 & 12.1 & & & & & & & 0.0 & 5.5 & 0.0 & 2.5 & 0.0 & 1.1 & 0.0 & 0.5 & 0.0 & 0.2 & 0.0 & 0.1 \\
\hline \multirow[t]{2}{*}{5} & 1 & 40.7 & & & & & & & & & 17.4 & 0.9 & 7.4 & 0.8 & 3.2 & 0.5 & 1.4 & 0.3 & 0.6 & 0.2 \\
\hline & 2 & 14.3 & & & & & & & & & 0.0 & 6.4 & 0.0 & 2.9 & 0.0 & 1.3 & 0.0 & 0.6 & 0.0 & 0.3 \\
\hline \multirow[t]{2}{*}{6} & 1 & 38.7 & & & & & & & & & & & 16.5 & 0.9 & 7.1 & 0.8 & 3.0 & 0.5 & 1.3 & 0.3 \\
\hline & 2 & 16.3 & & & & & & & & & & & 0.0 & 7.3 & 0.0 & 3.3 & 0.0 & 1.5 & 0.0 & 0.7 \\
\hline \multirow[t]{2}{*}{7} & 1 & 36.8 & & & & & & & & & & & & & 15.7 & 0.8 & 6.7 & 0.7 & 2.9 & 0.5 \\
\hline & 2 & 18.2 & & & & & & & & & & & & & 0.0 & 8.2 & 0.0 & 3.7 & 0.0 & 1.7 \\
\hline \multirow[t]{2}{*}{8} & 1 & 34.9 & & & & & & & & & & & & & & & 14.9 & 0.8 & 6.4 & 0.7 \\
\hline & 2 & 20.1 & & & & & & & & & & & & & & & 0.0 & 9.0 & 0.0 & 4.1 \\
\hline \multirow[t]{2}{*}{9} & 1 & 33.2 & & & & & & & & & & & & & & & & & 14.2 & 0.7 \\
\hline & 2 & 21.8 & & & & & & & & & & & & & & & & & 0.0 & 9.8 \\
\hline
\end{tabular}

our prediction of population growth rate $\lambda$ by about $3 \%$ (see Section 1). For the expected $m$-array in Table 1 , the estimate of local emigration is 0.050 , with $95 \%$ confidence limits of 0.032 and 0.073 .

Figure 2 shows the results of repeating the above calculations, for a range of capture probabilities in the peripheral area and a range of probabilities of local emigration. The confidence limits have been plotted relative to the corresponding estimate, in order to put them on a common scale. In Figure 2a, it is clear that as the capture probability increases to 0.4 , the precision improves, with little benefit thereafter. Figure $2 \mathrm{~b}$ suggests a similar conclusion for the estimation of local emigration: a clear improvement in precision as the capture probability increases from 0.1 to 0.4 , but little benefit thereafter. Even with a ten-year study and a high capture probability in the peripheral area, the estimate of local emigration can be relatively imprecise.

\section{DISCUSSION}

The method we have presented here is designed to help researchers make decisions about the level of field effort required to assess the amount of local emigration from a study population. We have also presented a range of calculations for a particular example, but the approach can readily be adapted to other scenarios. For example, we chose to focus on a situation in which there is no movement from the peripheral area into the main area, that is, that local emigration is permanent. The extent to which the choice of study design might be 
affected by this assumption can be checked by considering alternative plausible values for this probability of movement. There is a link here with the work on estimating temporary emigration (random or otherwise) using Pollock's robust design (Kendall, Pollock, and Hines 1997). If any local emigration were temporary and field effort in the peripheral area were not feasible, use of the robust design could be considered.

a)

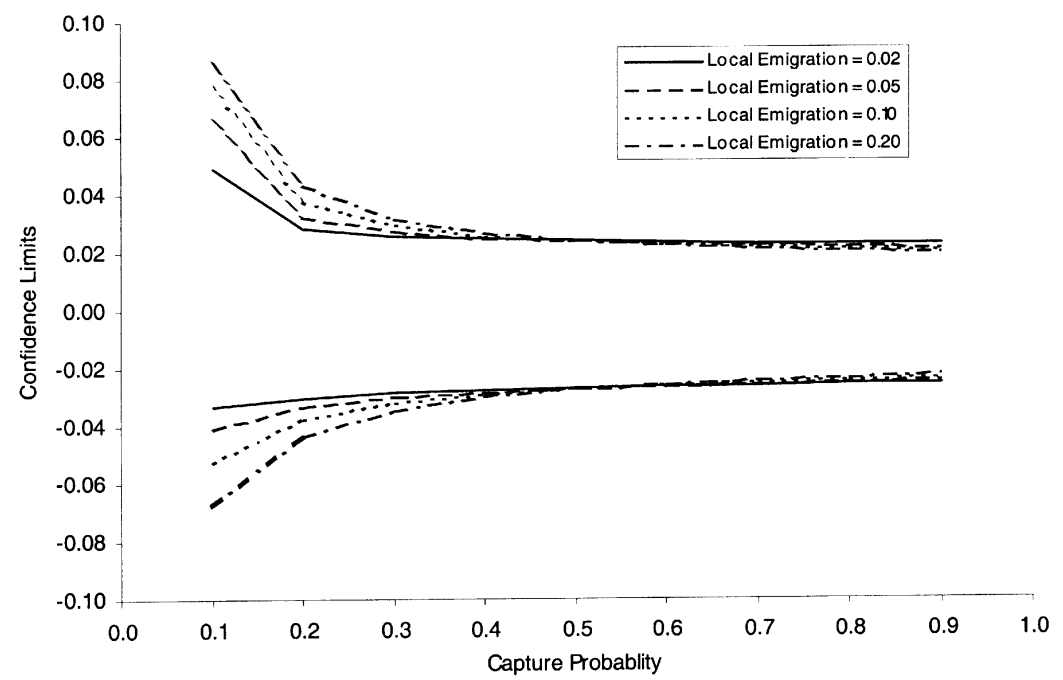

b)

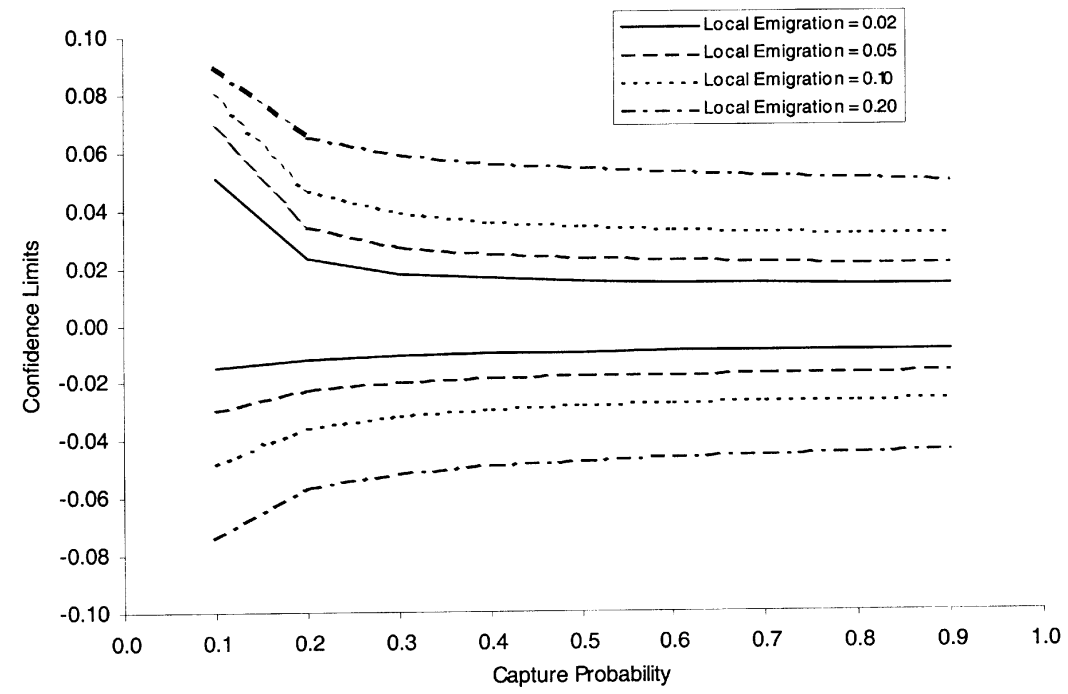

Figure 2. Expected 95\% profile likelihood confidence limits (relative to the estimate) for (a) survival probability and (b) probability of local emigration, versus capture probability in the peripheral area, for a study over ten years and for a range of probabilities of local emigration. Survival probability $=0.9$, capture probability in main area $=0.5$, initial population size in main area $=100$, initial population size in peripheral area $=10$. 
It is important to emphasize that emigration (permanent or temporary) is not the only possible source of bias in our estimate of survival probability, heterogeneity of capture probability being the most commonly considered (Carothers 1973).

As in the design of any mark-recapture study, a practical difficulty is the need to specify the population sizes at the beginning of the study. In using the methods presented here, it would be sensible to consider the robustness of the conclusions about the design of the study to specification of the population sizes in the two areas.

It may also be worth considering the extent to which the conclusions depend upon the model that is assumed in both generating the expected $m$-array and then calculating the confidence limits. We chose a relatively simple, time-independent model here, in the hope that our choice of design would not be greatly different for other models. Such a model involves the biologically unrealistic assumption that survival probability does not change during the study. A topic for further research would be the extent to which the choice of design would change if we were to use a random effects model (White and Burnham 1999).

\section{ACKNOWLEDGMENTS}

We are grateful to two anonymous referees for their comments, which helped improve the presentation of the article.

[Received June 2001. Revised December 2001.]

\section{REFERENCES}

Arnason, A. N. (1972), "Parameter Estimates from Mark-Recapture Experiments on Two Populations Subject to Migration and Death," Researches on Population Ecology, 13, 97-113.

_ (1973), "The Estimation of Population Size, Migration Rates, and Survival in a Stratified Population," Researches on Population Ecology, 15, 1-8.

Brownie, C., Hines, J. E., Nichols, J. D., Pollock, K. H., and Hestbeck, J. B. (1993), “Capture-Recapture Studies for Multiple State Including Non-Markovian Transitions," Biometrics, 49, 1173-1187.

Burnham, K. P., Anderson, D. R., White, G. C., Brownie, C., and Pollock, K. H. (1987), "Design and Analysis Methods for Fish Survival Experiments Based on Release-Recapture," American Fisheries Society Monograph 5.

Cam, E., Hines, J. E., Monnat, J.-Y., Nichols, J. D., and Danchin, E. (1998), "Are Adult Non-breeders Prudent Parents? The Kittiwake Model," Ecology, 79, 2917-2930.

Cameron, C., Barker, R., Fletcher, D., Slooten, E., and Dawson, S. (1999), "Modelling Survival of Hector's Dolphins Around Banks Peninsula, New Zealand," Journal of Agricultural, Biological, and Environmental Statistics, 4, 1-10.

Carothers, A. D. (1973), "The Effects of Unequal Catchability on Jolly-Seber Estimates," Biometrics, 29, 79-100.

Dawson, S. M., and Slooten, E. (1993), "Conservation of Hector's Dolphins, The Case and Process Which led to the Establishment of the Banks Peninsula Marine Mammal Sanctuary," Aquatic Conservation, 3, 207-221.

Heppell, S. S., Caswell, H., and Crowder, L. B. (2000), "Life Histories and Elasticity Patterns, Perturbation Analysis for Species with Minimal Demographic Data," Ecology, 81, 654-665. 
Hestbeck, J. B., Nichols, J. D., and Malecki, R.A. (1991), "Estimates of Movement and Site Fidelity Using Mark-Resight Data of Wintering Canada Geese," Ecology, 72, 523-533.

Kendall, W. L., Nichols, J. D., and Hines, J. E. (1997), "Estimating Temporary Emigration Using CaptureRecapture Data with Pollock's Robust Design,” Ecology, 78, 563-578.

Lebreton, J-D., Burnham, K. P., Clobert, J., and Anderson, D.R. (1992), "Modelling Survival and Testing Biological Hypotheses Sing Marked Animals, A Unified Approach with Case Studies," Ecological Monographs, 62, 67-118.

McCullagh, P., and Nelder, J. A. (1989), Generalized Linear Models (2nd ed.), London: Chapman and Hall.

Nichols, J. D., and Kendall, W. L. (1995), “The Use of Multi-state Capture-Recapture Models to Address Questions in Evolutionary Ecology," Journal of Applied Statistics, 22, 835-846.

Pollock, K. H., Nichols, J. D., Brownie, C., and Hines, J. E. (1990), "Statistical Inference for Capture-Recapture Experiments," Wildlife Monographs, 107, 1-97.

Schwarz, C. J., Schweigert, J. F., and Arnason, A. N. (1993), "Estimating Migration Rates Using Tag-Recovery Data," Biometrics, 49, 177-193.

Scofield, P., Fletcher, D. J., and Robertson, C. (2001), "Titi (Sooty Shearwaters) on Whero Island, An Analysis of Historic Banding Data Using Modern Capture-Recapture Techniques," Journal of Agricultural, Biological, and Environmental Statistics, 6, 268-280.

Slooten, E., Dawson, S. M., and Lad, F. (1992), "Survival Rates of Photographically Identified Hector's Dolphins from 1984 to 1988," Marine Mammal Science, 8, 327-343.

White, G. C., and Burnham, K. P. (1999), "Program MARK, Survival Estimation from Populations of Marked Animals," Bird Study, 46 Supplement, 120-138. 